\title{
SPINAL CORD STIMULATION: FIBER DIAMETERS IN THE DORSAL COLUMNS MODELED FROM CLINICAL DATA
}

\author{
Wilbert A. Wesselink, Jan Holsheimer \\ Institute for Biomedical Technology, University of Twente \\ P. O. Box 217, 7500 AE, Enschede, The Netherlands \\ email: w.a.wesselink@el.utwente.nl
}

\begin{abstract}
Computer simulations of clinical data were performed to estimate the diameter distribution of $A \alpha \beta$ nerve fibers in the human dorsal colums, activated by spinal cord stimulation. Qualitatively, the calculated distribution was in accordance with experimental data. Due to mismatches in impedance and limitated resolution of the $C T$ scans more patient data is needed to reliably predict the quantitative diameter distribution.
\end{abstract}

\section{INTRODUCTION}

Spinal cord stimulation (SCS) for the management of chronic pain is based on the 'Gate Control' theory. It is assumed that the inhibition of small diameter noxious pathways is modulated by activation of large diameter sensory $(A \alpha \beta)$ nerve fibers $(7-15 \mu \mathrm{m})$. Suppression of chronic pain can be achieved by stimulation of these large diameter fibers in the dorsal columns of the spinal cord. It is accompanied by paresthesia, which may cover those dermatomes corresponding to the spinal cord level of the cathode down to the caudal end.

To achieve a better understanding of SCS, a computer model has becn developed at the University of Twente (UTSCS model) [1]. With the UT-SCS model it is possible to simulate SCS by epidurally placed electrodes. For some years, the model has been used for the development of new electrodes, which may give better results the paresthesia coverage of painfull areas and a decrease of unwanted side effects.

Although most of the essential model parameters are known fairly well from literature, the mediolateral diameter distribution of the $A \alpha \beta$ fibers in the human dorsal columns is still unknown. A diameter reduction from a lateral to a medial fiber position in expected [2]. The recruitment order of paresthesia areas is related to the diameters and the positions of the corresponding fibers in the dorsal columns. In order to predict this recruitment order, it is essential to know the fiber diameter distribution in the superficial dorsal column layers

In this study, the diameter distribution of the large diameter sensory nerve fibers in at the border of the human dorsal columns was estimated by modeling clinical data, i.e. the actual geometry of a patients spinal cord, the recruitment order of the paresthesia areas, and the voltage levels at which the corresponding dorsal column fibers are excited.

\section{METHOOS}

Clinical data were obtained from patients enrolled in a study to test a new multi contact electrode for SCS [3]. Patient selection, implantation of the electrode, and patient testing were performed at the University Hospital 'Gasthuisberg' in Leuven, Belgium. Transverse CT-scans of the spinal cord were made at the level of implantation. before and after surgery. Subsequently, several test sessions with the patient were performed. During these sessions, the patients described the distribution of paresthesia as a function of voltage level during stimulation with several anode-cathode combinations. Impedances were measured as well.

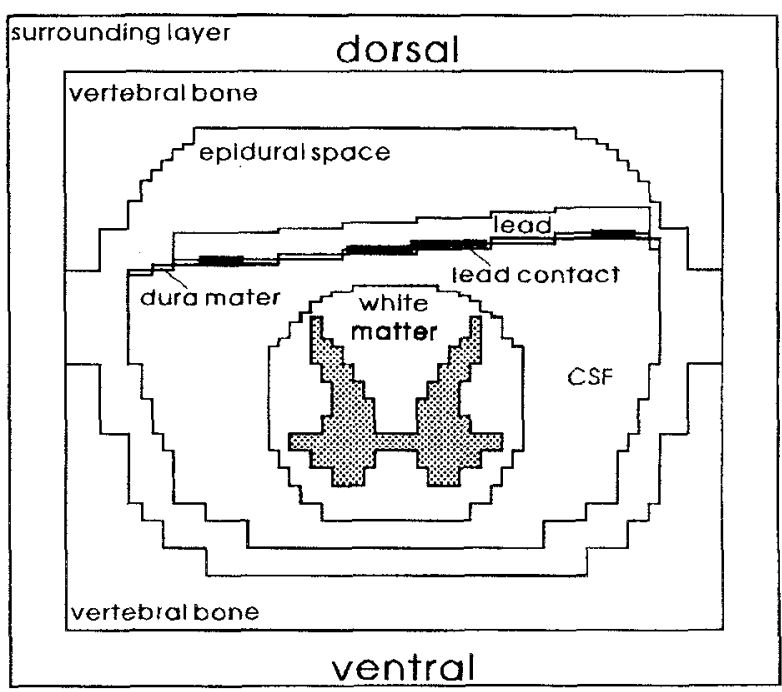

Figure 1: Transverse section of the spinal model at 19

The transverse CT-scans of a patient's spinal cord were used to make accurate discretized $3 \mathrm{D}$ reconstruction with the 
UT-SCS model. Figure 1 shows a transverse section of a patient's spinal cord model at level T9. This inhomogeneous volume conductor model represents the gross anatomy of the spinal cord, e.g gray and white matter, epidural space, dura mater, vertebral bone, cerebrospinal fluid (CSF) and a layer representing surrounding tissues. These anatomical structures are characterized by their electrical conductivities. After realization of the $3 \mathrm{D}$ volume conductor model, the potential field, induced by the epidural lead contacts, was calculated by solving the discretized Laplace equation using a Red-Black Gauss-Seidel iteration with variable overrelaxation [4].

Hereafter, the calculated potential field was used to determine the response of the branched nerve fibers at the border of the dorsal columns. The electrical behaviour of these myelinated dorsal column (DC) fibers is described with a McNeal type model. Parameters of the nodal membrane were taken from experimental data from Chiu et al. |11. As in the clinical situation, threshold voltages for excitation of the considered DC fibers were determined using a rectangular pulse with a duration of $210 \mu \mathrm{s}$.

According to neuroanatomical data the positions of the DC fibers at level T9 corresponding to the different paresthesia areas from medially to laterally in the dorsal columns were considered to be: posterior thigh, posterior calf, foot anterior calf, anterior thigh, and groin. The distance between two consecutive fibers increased from medial to lateral.

\section{RESULTS}

The results presented here were calculated from a patient test, where three contact combinations were used, i.e. a monopole, a longitudinal bipole, and a transverse tripole (central cathode, lateral anodes). In figure 1, it is shown that in this patient the electrode was placed slightly asymmetrical and at a distance of $1 \mathrm{~mm}$ relative to the spinal cord

Figure 2 shows the diameter distribution as calculated for this patient. Triangles represent results of the monopole, squares the results of the bipole and the results of the tripole are represented by crosses. As can be seen, the medial DC fibers were not stimulated by the monopolar configuration. The fiber diameters range from 2 to $6 \mu \mathrm{m}$, which is smaller than expected. Except for the fibers corresponding to the left posterior calf and thigh, both sides show an increase in diameter in the lateral direction, which is in accordance with the trend as found in cat by Petit and Burgess [2].

When the measured and calculated impedances of the considered contact combinations were compared, it appeared that the model overestimated these impedances by approximately a factor 2 . This might be caused by an underestimation of the modeled dura mater conductivity.

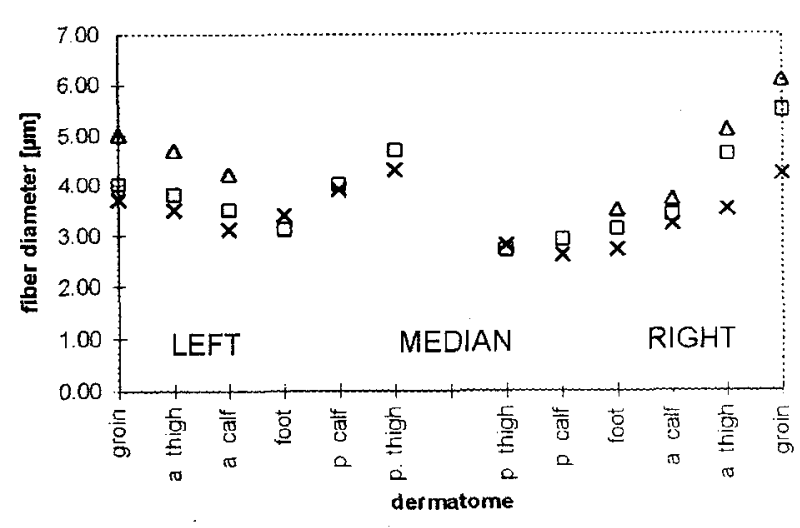

Figure 2: Calculated fiber diameter distribution at the dorsal column border

\section{DISCUSSION}

With clinical data as the input for the UT-SCS computer model it is possible to estimate the diameter distribution of the nerve fibers at the dorsal column border of the human spinal cord. However, the presented example shows that the model calculated impedances approximately 2 times too high. As a consequence, the fiber diameters would even be smaller than calculated here. If it is assumed that the $A \alpha \beta$ fibers are not present at the border, but slightly deeper in the dorsal columns, larger fiber diameters will be calculated.

The larger diameters at the left median dorsal column might be caused by a local anatomical irregularity, e.g. a thicker pia mater. Due to the limited resolution of the CTscans, such details are invisible.

Further analysis and more patient data are needed to reliably predict the diameter distribution of the DC fibers along the superficial dorsal columns.

Acknowledgement: This study was supported by a grant from Medtronic, Minneapolis.

\section{REFERENCES}

[1] J.J. Struijk, J. Holsheimer, G.G. v.d. Heide, H.B.K. Boom, Recruitment of dorsal column fibers in spinal cord stimulation: Influence of collateral branching, IEEE Trans, on Biomed. Eng., vol. 39. pp 903. 912,1992

[2] D. Petit, P.R. Burgess, Dorsal column projection of receptors in cat hairy skin supplied by myelinated fibers, J. Neurophys. Sci., vol.27, pp. 163-172, 1976

[3] J.J. Struijk, J. Holsheimer, G. Barolat, J. He, H.B.K. Boom, Paresthesia thresholds in spinal cord stimulation: A comparison of theoretical results with clinical data. IELE Trans. Rehab. Ing., vol. 1. pp 101-108, 1993

[4] J.J. Struijk, J. Holsheimer, Transverse tripolar spinal cord stimulation: theoretical performance of a dual channel system, Med. Biol. Eng. Comp. (in press) 